\title{
Review of Complex Networks
}

\author{
Ben-Bright Benuwa \\ School of Computer Science \\ Data Link Institute \\ P. O Box 2481, Tema
}

\author{
Benjamin Ghansah, PhD \\ School of Computer Science \\ Data Link Institute \\ P. O Box 2481, Tema
}

\author{
Dickson Keddy Wornyo \\ School of Computer Science \\ Data Link Institute \\ P. O Box 2481, Tema
}

\begin{abstract}
Research into complex networks $(\mathrm{CN})$ has become a key topic of interest across many disciplines in recent times. Complex systems are naturally pervasive and man-made systems, and because $\mathrm{CN}$ could be regarded as the bedrock of complex systems they appear in a wide range of scenarios stretching from societal and environmental to biological and technological systems. Stimulated by experimental and pragmatic analysis of interacted or connected systems such as the Internet, biological, and social networks, proponents in recent times have advanced diversified approaches and frameworks to aid in envisaging the performance of these systems. This paper presents a review in the field of $\mathrm{CN}$, in particular, the outsets of degree distributions, the small-world effect, network correlations, clustering, synchronization, random graph models, models of network growth and special attachment, robustness and dynamical processes- interestingly taking place on networks. This review also aims at serving as a substratum for future research in the study area, particularly those pursuing their career synthetically in artificial intelligence. In the end, some important conclusions and possible research directions of $\mathrm{CN}$ that essentially need to be studied in the future are proposed.
\end{abstract}

\section{Keywords}

Complex network, scale-free networks, small world networks, synchronization

\section{INTRODUCTION}

In the framework of network theory, a $\mathrm{CN}$ is a graph or network substantially with vital topological features that does not arise in simple networks such as lattices or random graphs but often occur in graphs modeling real world systems[1,2]. The study of $\mathrm{CN}$ is a novel and vigorous area of scientific research largely motivated by the empirical study of real-world networks such as the social, biological and, computer networks. More so most of these networks display substantial amount of important topological features, with forms of connections between the elements that are not purely regular and purely random [3]. These features similarly comprise of mutuality, triad importance profile and other features in the instance of directed networks[4, 5]. On the contrary, a lot of the mathematical representations of the past studied networks such as lattices and random graphs, do not exhibit these features[5]. The utmost complex frameworks could be recognized by networks with a moderate amount of interactions[6]. This relates to the circumstance that the maximum information content (entropy) is obtained for medium probabilities.

There are two eminent and considerably learnt classes of $\mathrm{CN}$. These are scale-free networks [7] and small-world networks[8, 9], whose discovery and characterization are recognized case studies in the area under consideration. Both networks considerably have some explicit structural features; the power-law degree distributions with respect to the former and highly clustered, like regular lattices, hitherto have small distinctive characteristic path lengths, like random graphs for the latter[10]. Moreover, there has been a developing concern by many researchers in other facets of the structures of these networks as the learning of $\mathrm{CN}$ continues to grow in popularity and significance [11] as a result of the proliferation of social network..

More recently, the study of $\mathrm{CN}$ has been extended to networks of networks [12] and if those networks are codependent, they develop considerably to be more susceptible to haphazard failures and targeted attacks and exhibit cascading failures and first-order percolation evolutions [13].

The field continues to progressively grow at a fast pace, and has brought together researchers from many areas including mathematics,

physics, biology, telecommunications, computer

science, sociology, epidemiology, and others [14]. Ideas as a result of network science and engineering remained realistically useful to the analysis of genetic regulatory and metabolic networks; the modeling and design of scalable communication networks such as the conception and generation of complex wireless networks [15]; the formulation of vaccination stratagems for the regulation of disease; and a wide array of other practical issues. There has been a lot of research and regular publication on the field networks in some of the best noticeable scientific journals and vigorous financial backings in lot countries. It has been the topic of many conferences in a diversity of different fields, and has been the subject of several books both for the lay person and for the expert.

\section{SCALE FREE NETWORKS}

A network is termed scale-free [7] if its degree distribution, thus, the likelihood that a node chosen homogenously at random has a definite number of degree (links), obeys a specific mathematical function called a power law. The power law suggests that the degree distribution of these networks has no characteristic scale. In contrast, networks with a solitary distinct scale are slightly comparable to a lattice suggesting that every single node roughly has the identical degree. Examples of networks with a solitary scale include the ErdösRényi (ER) random graph [16, 17]and hypercubes[18]. In a scale-free degree distribution networks, some vertices would have a degree with their orders of enormousness grander than the average and are mostly mentioned as hubs, although this maybe a bit ambiguous as there is no intrinsic threshold above which a node could be understood as a hub. If there were any such threshold, the network would not have been thought of as a scale-free[19].

An increasing interest in scale-free networks started in the early years which were allied with the reporting of discoveries of power-law degree distributions in everyday life networks such as the World Wide Web, the network of Autonomous 
systems (ASs), protein collaboration networks, networks of Internet routers (some), email networks, and many more. However, most of these power laws fell prey when subjected to laborious statistical testing, but many of the networks generally genuinely exhibits the more general idea of heavytailed degree distributions before finite-size effects occur and are very diverse from what one would expect if edges existed autonomously and at random (i.e., if they followed a Poisson distribution). There are a number of ways for building networks with power-law degree distribution. The Yule process for instance is an established reproductive process for power laws, and has been acknowledged since 1925 . However, it has by a lot of other names, some of which are The Gibrat principle by Herbert A. Simon, the Matthew effect, cumulative advantage and, preferential attachment by Barabási and Albert for power-law degree distributions. Recently, Hyperbolic Geometric Graphs have alternatively been proposed as hitherto another way of building scale-free networks as a result of its recurrent reinvention.

Some networks connected with a power-law degree distribution and specific supplementary types of structure can be highly resistant to the random removal of vertices hence the vast majority of vertices remain connected together in a gigantic constituent [20]. These networks could be prone and liable to beset attacks intended at shattering the network rapidly. The critical vertices, the ones linked with the utmost degree, normally linked with the spread of disease either natural or artificial and are responsible for the spread of fads with regards to communication and social networks, when the graph is homogeneously or regularly random apart from the degree distribution. While random graphs have a distance averaging of order $\log \mathrm{N}[8]$ between nodes, with $\mathrm{N}$ being the number of nodes, scale free graph could have a distance of log $\log \mathrm{N}$ and are referred to as an ultra-small world networks [21]. According to [11] certain nodes maybe more vastly coupled than others in any real world networks and to measure this outcome, we could assume $p k$ to represent the segment of nodes that have $d$ links. Here $k$ is known as the degree and $p k$ is the degree distribution.

The simplest random graph replicas [22, 23] envisage a bellshaped Poisson distribution for $p k$ which is extremely lopsided and falloffs much more sluggishly than a Poisson for a lot of real world networks [11]. For example, the distribution decays as a power law $p k \sim k^{-y}$ for the Internet backbone [24], metabolic reaction networks [25], the telephone call graph [26] and the World-Wide Web [27] (Fig. 1a). In reality, there are a diminutive nodes with many links and have been dubbed 'scale-free'[28] [29] (Fig. 1d).

The presence of two-point connectivity correlations does not extremely change the flaw for Scale-Free networks to prevalent distribution and this result is linked to the deviation of the nearest neighbors average connectivity, which is guaranteed by the connectivity comprehensive balance condition, to be satisfied in physical networks [30]

The scale-free property is common but not universal [31]. The $p k$ fits better for a power law by way of an exponential cutoff [32] in coauthorship networks (Fig. 1b); it is an exponential distribution in the power grid of the western US [31] (Fig. 1c); and is Gaussian [31] (Fig. 1d) in the social network of Mormons in Utah [33].
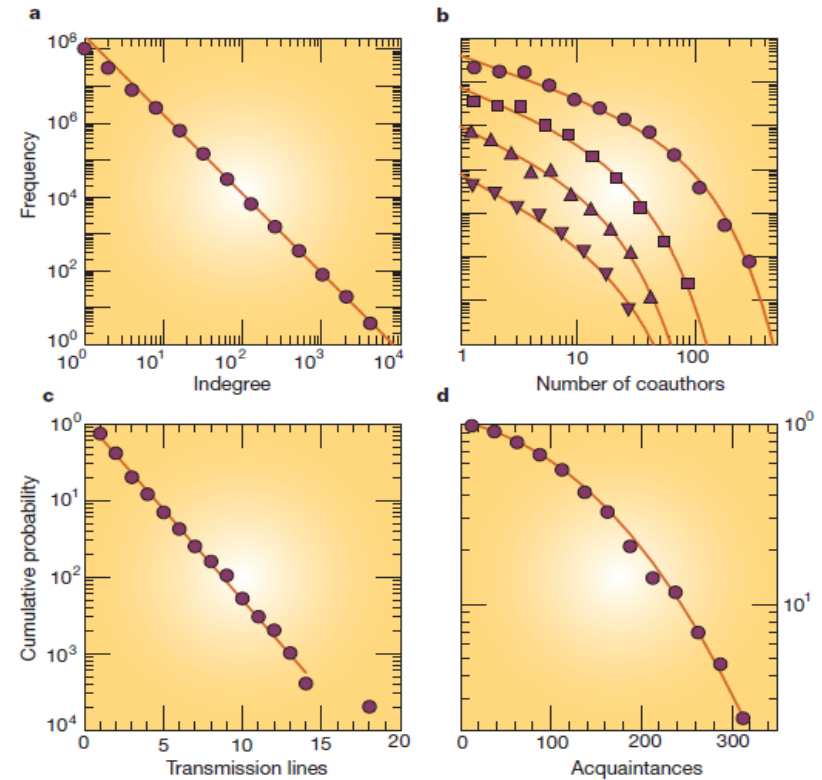

Figure 1: Graph of Coauthorship networks

\section{SMALL-WORLD NETWORKS}

A network is christened small-world [8] if by resemblance it exhibits the properties associated with the small-world phenomenon, and are generally known as six degrees of separation. The theory of small world, foremost defined by [34], and experimentally tested by [35], is the notion that two individuals are linked by just six degrees of separation unsystematically, i.e. the diameter of the corresponding graph of social connections is not much larger than six. [8] Published the foremost small-world network ideal that interposes effortlessly by single parameter amongst a random graph and a lattice. Their model established that with the inclusion of just a few numeral of long ranged links, a regular graph, in which the diameter is relatively proportionate to the magnitude of the network, can be converted into a "small world" in which the average numeral of edges among some two vertices is very small expected to grow statistically as the logarithm of the size of the network, with clustering coefficient persistently being large. It is believed that real world networks such as the World Wide Web and the metabolic network and also a percentage of abstract graphs such as random graphs and scale-free networks portray the small-world property. There is an doubt connected with the term "small world" with respect to the scientific literature on networks, and in accordance with mentioning the size of the diameter of the network, it could also be seen as the cooccurrence of a small diameter and a high clustering coefficient. The clustering coefficient is a measure that denotes the concentration of triangles in the network. For example, sparse random graphs diminishingly obligate a few clustering coefficient. However real-world networks often have a coefficient expressively higher and this disparity is attributed to the fact that edges are correlated in real world networks as argued by Scientists.

Research has shown that, high-speed communication channels could be offered by short paths among aloof parts of the system, thereby enabling dynamical processes such as synchronous harmonization that necessitates information flow and coordination universally. Further research that proceeded along countless fronts and several experimental applications of small-world networks have been carried out and also 
documented, in areas stretching from business to cell biology $[25,31,32,36-40]$. Furthermore, the small-world networks are found to be theoretically behaving like Rorschach test in which different scientists have dissimilar methodologies to problems subject to their disciplines or areas of study. For instance, computer scientists are usually faced with problems about algorithms and their complexity. [11] Indicated that graphs linked with a lot difficult search issues have a smallworld topology. [41] introduced a well-designed model of the algorithmic problem revealed by Milgram's original sociological experiment[35]on ways of identifying a short chain of acquaintances by linking yourself to an arbitrary target person, utilizing local information only, and he ascertained that the problem is effortlessly resolvable for certain categories of small worlds, and fundamentally intractable for others.

Clustering locally and global exchanges could together affect the spread of contagious diseases, with consequences for vaccination strategies and the advancement of virulence [4245]. Neurobiologists have debated severally on the possible evolutionary importance of small-world neural topology that combines fast signal processing with coherent oscillations [46], and appeared neither like regular nor random architectures, and could be chosen by getting used to ironically sensual surroundings and motor demands [39]. Perchance the strongest response to the Rorschach experiment could have originated from the statistical physicists, who recognized immediately [47] that the toy model of [8] would yield to their techniques[48]. In its enhanced form the model begins with a circle of $n$ nodes linked by an undirected acquaintances to its closest and next closest neighbors up to a certain range $k$. Shortcut connections are introduced amongst randomly designated pairs of nodes, with a probability $(p)$ per connection on the fundamental lattice instead of being rewired; accordingly there exist naturally $n k p$ shortcuts in the graph (Fig. 2). The question is: on average, to move between nodes, how many steps are needed to enroot the shortest path? If the average separation is denoted by $l$, it could be found that $l$ falls abruptly close to $p=0$, endorsing that few shortcuts do undeniably constrict the universe melodramatically and as derived by [49] in the formulation below:

$l=\frac{n}{k} f(n k p)$

Where

$f(x)=\frac{1}{2 \sqrt{x^{2}+2 x}} \tanh ^{-1} \frac{x}{\sqrt{x^{2}+2 x}}$

This solution is asymptotically precise in the limits $n=\propto$ (large system size) and either $n k \mathrm{p}=\propto$ or $n k \mathrm{p}=0$ (large or few numerals of shortcuts). Figure 5 show that it also gives the correct qualitative behaviour for $n k p » 1$. [50] Enhanced this outcome by demonstrating a laborious distributional approximation for $\mathrm{p}$, sideways with a bound on the error.

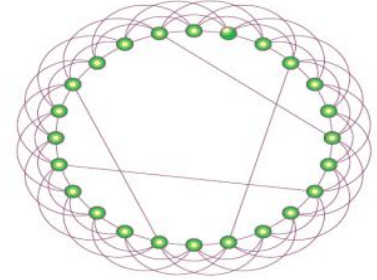

Figure 2 Resolvable model of a small-world network [49].

\section{UNDERSTANDING THE METRIC OF COMPLEX NETWORK}

A graph, denoted by $G$, consisting of a collection $N$ of $N$ nodes connected by a collection $L$ of $L$ links could be used to represent the topology of $\mathrm{CN}$. Graphs with $\mathrm{N}$ nodes, in turn, are completely described as an $N \times N$ of adjacency matrix T, in which the entities or components $t_{i j}=1$ if there exist a link between node $i$ and node $j$, otherwise $t_{i j}=0$. All entities $t_{i j}$ of the adjacency matrix $\mathrm{T}$ are consequently either 1 or 0 and $\mathrm{T}$ is symmetrical for undirected graphs, and unsymmetrical for directed graphs, where there could be a link from $\mathrm{i} \rightarrow \mathrm{j}$, but not in the contradictory bearing $\mathrm{j} \rightarrow \mathrm{i}$. Undirected graphs are considerably easier to explore because the symmetry of $\mathrm{T}$ agreeably results in a prevailing assets such as real eigenvalues which are mostly complex with respect to nonsymmetric matrix and eigenvectors. The importance of links in $\mathrm{CN}$ varies from one link to the other and weights could be associated to links to distinguish some added assets such as cost, distance, capacity, and structure amongst other things. The matrix $\mathrm{T}$, assumes the same structure of the weighted adjacency matrix $P$, just that the element $P_{i j}$ is a real number, that typifies some assets of the link[51]. The assembly of all link weights associated with a graph is called the link weight structure. Surprisingly, there are basically few networks for which this link weight structure, for instance the distances in transportation networks is known, but the sum of money transfer among banks, the delay in the Internet, the strength of the interaction in the human brain, reaction quantifiers in metabolic networks, and many more, may are/maybe difficult to realized.

Topological graph measurements such as the link betweenness, the nodes degree, the shortest path from I to j; hopcount, the clustering coefficient, the edge/link connectivity, etc could be computed from adjacency matrix [51-53]. Another type of metrics are spectral metrics, whose interest instigates from the point that the eigenvalues of a graph are a unique fingerprint $[54,55]$ such as the spectral gap, the biggest eigenvalue of the adjacency matrix; spectral radius, the algebraic connectivity, etc. the spectral domain consisting of the eigenvectors and eigenvalues of the adjacency matrix $T$ possesses the same information as the topology domain specified by $\mathrm{T}$. However, in disparity to topological measurements, spectral metrics need further mathematics and lack the provision of intuition[56].

Dynamics Of Complex Networks

The processes of network constitute the core of $\mathrm{CN}$ aside its topological structure. They tell why the network is constructed or created and they give value to a complex network. Examples of such processes or services include the transfer of IP packets in the instance of the Internet, the communication between functional regions of the brain, the blowout of rumors and news in a social network, and the transport of cars in a road network. In most cases, there are concerned in knowing whether the procedure is stable, phasetransitions [57] or forms of self-organization occur and how it behaves when the network grows (scaling laws) or is modified (removal or adding of subgraphs). The virus spread [58] and synchronization [59, 60] are reasonably well-understood examples.

Another difficult issue is the learning of the dealings among the processes on the network and the core network itself. For instance, when there exist a viral attack on a network, the only protection against it would be the installation of anti-virus software in the situation of computer networks or of vaccinations or medications with respect to the human social 
network. These movements do not alter the core topology. However, regulating the topology by sidestepping contact with infected nodes (e.g. computers or humans) brings about another type of protection that requires the comprehension of the coupling between graph and process (or function of the complex network)[56]. The last type of dynamics also obtains progressively more attention as many of the infrastructures are coupled [12]. For example, nearly all $\mathrm{CN}$ need energy, while the effect of digital communication to control these infrastructures increases. A failure occurring in the electricity distribution or in the control communication network may bring about failures or unexpected behavior in the functioning of the complex network whose torrent effects are not well understood.

More so, another theme is to comprehend how biological processes in nature attain such an amazing adaptively and resilience against external factors. For example, the Alzheimer disease is only diagnosed with certainty when over $80 \%$ of the links in the brains are destroyed [56].

\section{Robustness of Complex Networks}

One needs to be sure or convinced that the network is suitable or good with concerns to the issues that is essential to be handled throughout the construction and maintenance of a $\mathrm{CN}$ for the reasons for which it was designed for as could be understood as in Fig. 3 below. For instance, an Internet service provider would like to know if the present state of his network is good or not same with a doctor who may want to know whether the functional network state of his patients' brain is normal. The above questions are ill-posed hence needs to be visibly detailed or defined suitably. The apprehensions highlighted above have constrained the computation of robustness value, R-value of a given network at a specific period of time that concerns the service and the topology to ascertain the goodness of a complex network. The $\mathrm{R}$-value is a performance pointer that is pertinent for the service and normalized to the interval $[0,1]$. Thus, $\mathrm{R}=0$ corresponds to absence of network "goodness" and $\mathrm{R}=1$ reflects perfect "goodness". An example of a performance measure is a graph metric such as the average hop count. One of the reasons for a network robustness framework is to recommend an approach that elucidates and computes an Rvalue that symbolizes a level of robustness and to interpret Rvalues[61]

The reckoning of the R-value could be an aspect of periodic routine or an event-triggered network maintenance/management operation. As could be noticed from Fig. 3 below; the current R-value is matched with the minimal desired one, $R_{T}$ and either the $R$-value is sufficient in which case that ceases from taking any counteractive action, or the R-value is too low, in which case a modification to advance the graph is essential. The second objective of a network robustness structure is to propose effective, possibly optimal policies as to how a graph could be amended to upsurge its R-value subject to some cost criterion[56].

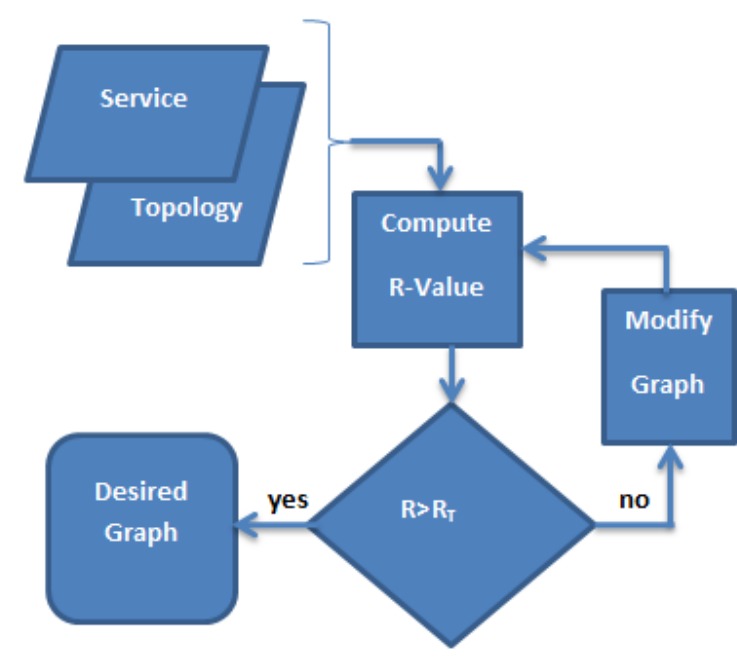

Figure 3: The flow chart of the high level goal to achieve network robustness [56].

Majority of graph metrics are not conjointly independent, and that their degree of correlation is graph specific [61]. Hence, collection of metrics alone is not adequate to differentiate them. Moreover, scaling a graph by increasing the numeral of nodes $\mathrm{N}$ or link $\mathrm{L}$ induces variations in the metrics, which brands it challenging to compare networks of different sizes.

A special branch of robustness is network security. In spite of the regular news reporting of intrusion and attacks of important computer systems, ranging from banks to governments, it is fair to mention that little systematic network theory is available to secure a network, aside some network immunization strategies[62, 63]. Most efforts today are heuristic, or unknown, since privacy and secrecy play an obscuring part in the domain of (network) security. A mammoth potential to employ the devices of complex network concept to design and construct supplementary secure networks, subject to confidentiality and low cost constraints, is lurking, but expertise appears lost.

\section{Synchronization In Complex Networks}

Synchronization procedures in populations of elements intermingling in the neighborhood are in the radar of intense research in chemical, physical, technological, biological, and social systems[64]. The countless efforts dedicated to understanding synchronization phenomena in natural systems have now taken pro of the present theory of $\mathrm{CN}$.

Synchronization was first studied mathematically (based on Fourier integrals) by $[65,66]$ who acknowledged its ubiquity in the natural world and speculated that it was associated in the generation of alpha rhythms in the brain but regrettably it fell on to it dead ends.

It is recognized that synchrony is entrenched in human life from the metabolic processes in the cells to the highest cognitive tasks performed as a group of individuals. For example, the outcome of synchrony has been labeled in experiments of individuals interacting, or at labor with an experience of collective, non-directive dialogue, rhythm or song, or of groups of kids interrelating to an insentient beat. The lack of such synchrony could result unconscious pressure, when goals could not be acknowledged nor achieved because the members are "out of sync" [67].

There are numerous capital workings that symbolized a revolution in the appreciation of these phenomena as segments of the struggles for the scientific account of 
synchronization phenomena. The architect of the pendulum clock learnt an unusual "kind of sympathy" in two pendulum clocks suspended side by side of each other and when swayed at 180 degrees out of phase with approximately the same frequency; the pendula were troubled, the anti-phase state was reinstated within 30mins and persisted indefinitely[68]. It was then presumed that, the critical communication for this result came from "imperceptible activities" of the usual frame backing up the dual clocks. From that time on, the phenomenon got into the focus of scientists. Synchronization encompasses, at least, two entities in communication, and the conduct of not many interacting oscillators has been extensively deliberated in mathematics and physics literature. Nonetheless, the phenomenon of synchronization of huge populaces is a dissimilar encounter and needs different hypothesis to be disentangled.

As stated in [64]Wiener did not make momentous mathematical advancement on the problem he modeled in his book"Cybernetics", nor did anybody till Winfree came along". [69]studied the dynamics of a huge population nonlinearly of feebly joined limit-cycle oscillators with inherent frequencies that were dispersed about certain mean value, based on some approved likelihood distribution. The breakthrough here was to cogitate the biological oscillators as phase oscillators, deserting the amplitude. Working within the structure of a mean field ideal, Winfree revealed that population of not identical oscillators could demonstrate an extraordinary accommodating phenomenon and huge variance of frequencies distribution may lead the oscillators incoherently running along each one with its natural frequency. This phenomenon relic when the variance is being reduced pending the span of an assured threshold. However, at a certain the threshold below, the oscillators activates to harmonize instinctively [70].

Even though Winfree's tactic evidenced to be fruitful in labeling the appearance of spur-of-the-moment order in the system, it was founded on the foundation that every oscillator senses the same array of connections.

However, this all-to-all connectivity among entities of a huge population is difficult to apprehend in the real-world. When the numeral of entities becomes adequately huge, the array is seen mismatched with physical constraints as may be the case of cost or energy minimization, and generally with the infrequent reflections on long ranged exchanges in systems designed by macroscopic entities. The precise native connectivity assembly of the entities was absent in these and subsequent approaches [64].

[64]introduced a modest prototypical of network arrangement, initially specifically anticipated to present the connectivity substrate in the problematic of harmonization or synchronization of cricket chirps, which showed a huge magnitude of harmonization over an elongated distances as if the insects were undetectably connected. Remarkably, this masterpiece did not seize as a new impact to synchronization or harmonization theory but instead the kernel for the contemporary theory of $\mathrm{CN}$ [8]. Furthermore, addition of small numeral of random connections starting with a regular lattice decreases the distance between nodes drastically, as depicted by Fig. 5 below. This feature, known as small-world effect, had been first reported in an experimentation piloted by [71] fact-finding the average path length with regards to social networks of individuals residing in the US. The analysis of harmonization procedures has not only profited from the improvement in the apprehension of the topology of $\mathrm{CN}$, but also its envelopment to the comprehension of general promising properties of networked systems. One objective that motivated this review paper is to revise precisely the exploration assumed as of now order to gain an insight into aftermath synchronization phenomena by the topological substrate of exchanges, of $\mathrm{CN}$.

$\dot{x}_{i}=f\left(x_{i}\right)+c \sum_{j=1}^{N} a_{i j} H\left(x_{j}\right) \quad x_{i} \in R^{n} \quad i=$

$1,2,3, \ldots, N$

(3)

Complete state synchronization:

$\lim _{t \rightarrow \infty}\left\|x_{i}(t)-x_{j}(t)\right\|_{2}=0, \quad i, j=1,2,3, \ldots, N$

State Synchronization:

$x_{1}(t) \rightarrow x_{2}(t) \rightarrow \cdots \rightarrow x_{N}(t) \rightarrow S(t)$

$\dot{S}(t)=f(s(t)) \quad$ (e.g. equilibrium point, periodic orbit, chaotic orbit)

$x_{1}=x_{2}=\cdots=x_{N}$ Synchronization manifold

$S(t) \in R^{n} \quad$ Synchronized state

Synchronization criteria

Eigenvalues of $\mathrm{L}=-\mathrm{A}: \quad 0=\lambda_{1}<\lambda_{2} \leq \cdots \leq \lambda_{N}$

Transversal subspace $(\perp$ synchronization manifold):

$$
\odot=\left(x=\left[x_{1}^{T}, x_{2}^{T}, \ldots, x_{N}^{T}\right]^{T} \in R^{n N}: \sum_{j-1}^{N} S_{j}^{t} x_{j}=0\right)
$$

Here, $\left[S_{1}^{t}, S_{2}^{t}, \ldots, S_{N}^{t}\right]^{T}$ is the left eigenvector of $\lambda_{1}=0$

Synchronization occurs if $c \lambda_{2}=0$ or if $\frac{\lambda_{N}}{\lambda_{2}} \in S_{2}$

As could be seen in figure 4 below; synchronization occurs at II and III where $S_{1}=\left(\alpha_{1}, \infty\right)$ and $S_{2}=\left(\alpha_{2}, \alpha_{3}\right)$. However synchronization did not happen at region I.

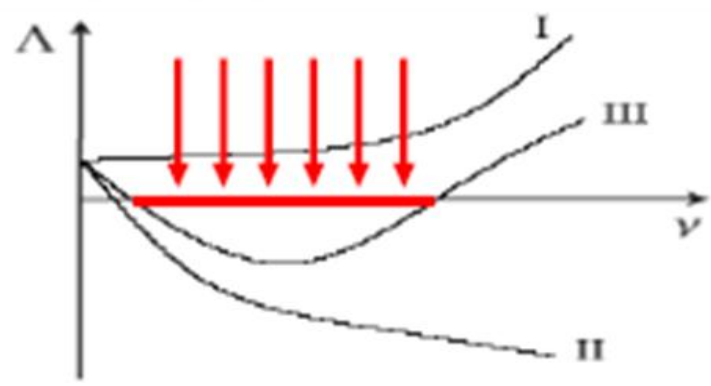

Figure 4

Synchronization in regularly combined or coupled networks is categorized into global and locally coupled networks. Globally coupled network will usually synchronize so long as the size is adequately large, no matter how small the combination strength c is. $\lambda_{2}=\cdots=\lambda_{N}=N$
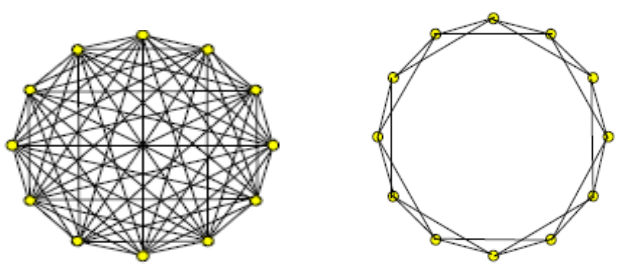

Figure 5: Small World Net [71] 
Locally coupled networks will usually not synchronize if its size is too large no matter how huge the combination strength $\mathrm{c}$ is.

$$
\lambda_{2}=4 \sum_{j=1}^{K / 2} \sin ^{2}(j \pi / N)
$$

Synchronization in small-world networks can greatly be achieved or enhanced by adding a tiny fraction of long-ranged connections to the construction of the regularly coupled locally network as revealed in fig 6 below.

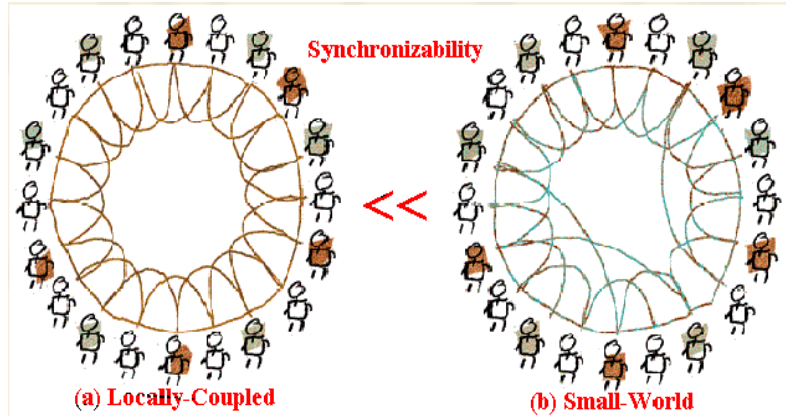

Figure 6: Synchronization in small world networks[72]

In scale-free networks, the principle of synchronization is about the same as that of the star-shaped network, mainly dogged by the one-to-one relations between the central node and each of the bordering or adjacent nodes. This is due to the extreme inhomogeneous connectivity distribution of scalefree networks which has few pivots which play a similar role as a solitary center in a star-shaped network as depicted by fig 7 below;

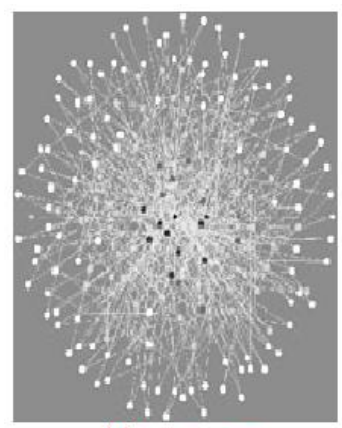

(a) Scale - Free

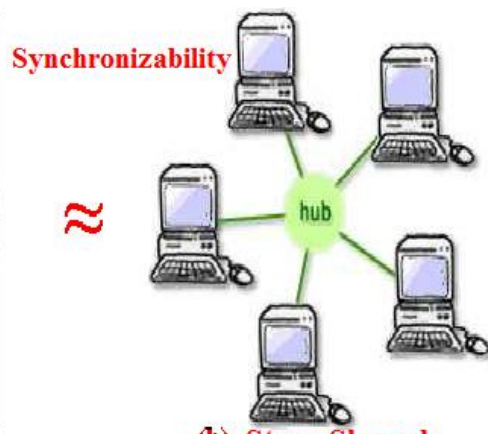

(b) Star-Shaped
Figure 7: Synchronization of SF networks [72]

The topology or the framework of the network also has an impact on synchronization. For instance, synchronization that exists in small-world networks increases as small world features increases. Larger clustering coefficient $(\mathrm{p}=1$, fully connected) implying smaller path length $\left(\lambda_{N} / \lambda_{2}\right)$ results in a better synchrony as revealed in fig. 8 (a) below. And in scalefree networks, synchronization increases as the power-law exponent increase with regards to the network topology as depicted by fig. (b) below; however, synchronization in both cases (scale-free networks and small-world) will also increase as their node betweenness decreases with respect to network topology therefore there is a strong association between betweenness and synchronization when dealing with homogeneous networks as in fig. 8 (c).
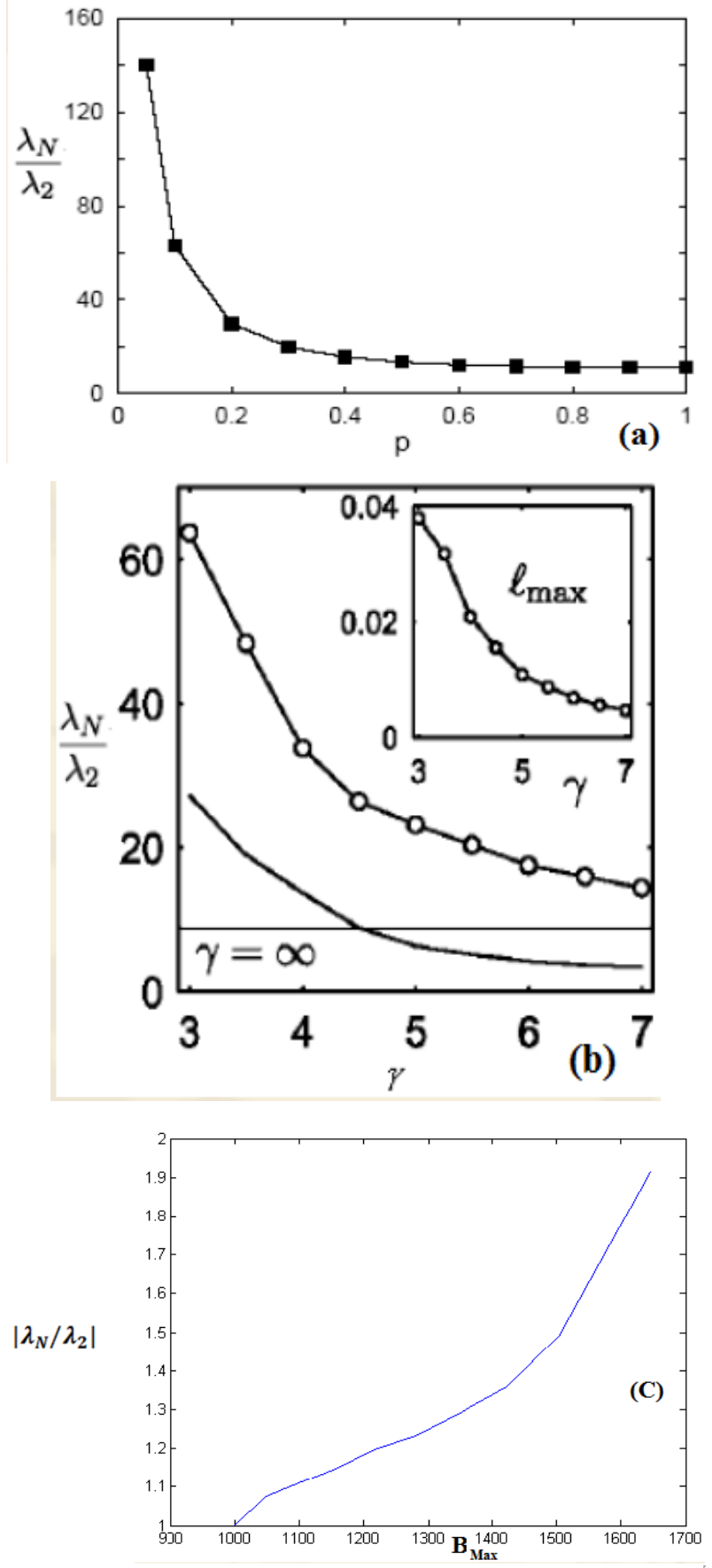

Figure 8

But in the heterogeneous networks, there is no vivid correlation or association between betweenness and synchronization as could be seen in figure 9 below. 


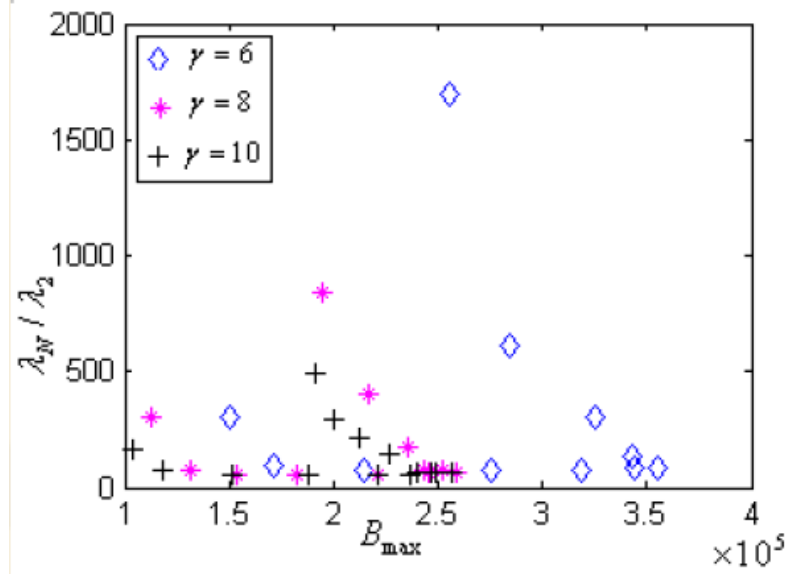

Figure 9: Graph of synchronization and betweeness [73]

The synchronizability of networks could be heightened by perturbing the structure or organization of the network to eliminate maximal betweenness [73] and also by modifying the coupling structure to reduce the influence of the heterogeneity of degree and betweenness distributions [74]. The node with largest betweenness is replaced with several connected nodes to be certain that the shortest path that passed through the original node will go through one or two new nodes which reduce the utmost betweenness dramatically. Edges with large loads can also (not only nodes with large betweenness) cause data traffic congestion, hence when such heavy loaded edges are also decoupled to redistribute the data traffic, the network becomes more efficient. This could be seen in fig 10 below;

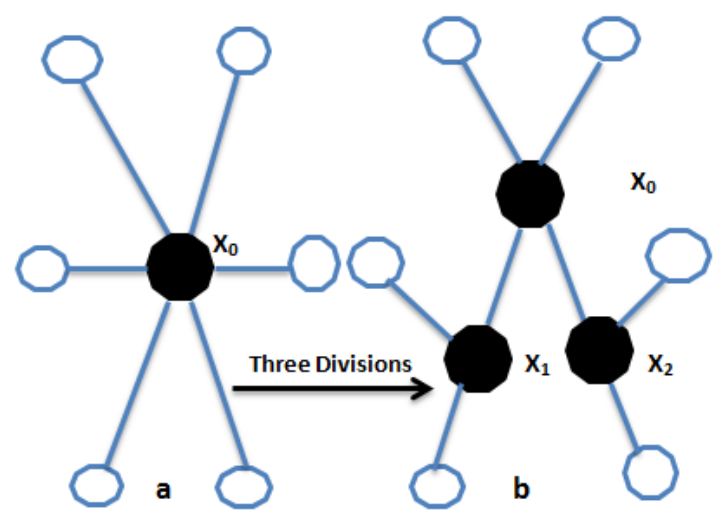

Figure 10: Synchronization through network structure perturbation [74]

\section{COMPLEX NETWORK ALGORITHMS AND COMPUTATIONS}

Awkwardly in $\mathrm{CN}$, most optimization issues usually exemplified in graphs are NP-hard, or polynomial hard which goes to propose that an optimal solution may not be computed for large $\mathrm{CN}$ and that heuristics is regularly the solitary alternative. In the instance of both virus spread and oscillators synchronization, the commencement of an infested network or coupling between oscillators is in reverse relational to the spectral radius $\lambda 1$ (A) of the graph. More so, for the amplification of the threshold, links or nodes could be removed. The elimination of those links that hugely lower the spectral radius or the least number of links to shift $\lambda 1$ (A) below some desired threshold is NP-hard [75]. The problem of searching for the best path subject to several constraints (such as distance, time and energy-consumption) is known as QoS-routing [76], and an optimal placement of regenerators in photosensitive networks [77] are both also NP-hard issues. For NP-complete problems, precise algorithms guzzle prohibitively long computation time so that heuristics are aimed to trade-off computation time for accuracy. Much effort has been fervent to heuristic algorithms that provide a solution that is guaranteed to not to further diverge than $\mathrm{x} \%$ of the universal optimum solution. Sometimes exact algorithms work surprisingly well in a certain kind of the state space [76]. Often constraints can be selected to enable efficient solutions [78].

Furthermore, another essential challenge in massive networks is the simple magnitude of its size, which averts that all details of the graph can be uploaded into a normal computer. Large complex network have stimulated the research on local computations, where only a certain surrounding of a node is taken into account, instead of the global topology information. Examples are "gossiping" algorithms [79], breath-first search of social networks and web-crawling. The massive growth in data and network sizes has headed to a fresh kind of "sublinear" algorithms that only consider part of the input and consequently run in sub-linear time complexity. Since not all information is considered, exactness could not be certain.

But through careful sampling, sub-linear algorithms might be capable to give acceptable bounds. Recently [80], some classical optimization problems have been approximated in sub-linear time.

Beside the classical distributed computation, where a large problem is split over several computers, a new flavor of this concept has been presented as "cloud-computing", where jobs are allotted to an amalgamation ("cloud") of servers and computing devices, utilized through a network.

\section{RELATED WORK AND APPLICATIONS OF COMPLEX NETWORKS}

The brain network, consisting of over $\mathrm{N}=10^{11}$ neurons and $\mathrm{L}$ $=10^{14}$ interconnections, with an estimated total of $500000 \mathrm{~km}$ wiring 6 is considered or seen as one of the most $\mathrm{CN}$. There are principally two core approaches in which complex network analysis can be engaged to understand the brain of humans [81], of which the functional brain unit is easier to measure. Understanding functional brain nets through topological measures such as degree, path length, clustering, assortativity, modularity [82] and/or by spectral metrics is still regarded as a sizzling topic of research. The applications this domain is massively huge: besides monitoring healthy people to understand emotion, memory and IQ [83], the major social and financial impact lies in understanding illnesses such as Alzheimer's disease and other categories of dementia, brain tumors [84] epilepsy and schizophrenia.

One of the recent applications of $\mathrm{CN}$ was a research conducted by [85] to discover whether Complex personal networks have any relationship with massive cognitive operative in the aged. Tougher involvement of the aged in social events or undertakings and grander entrenchments in networks is mostly contended to cushion or safeguard cognitive deterioration and lesser threats of dementia. It is believed that, when older people interact with other people the brain trained thereby enhancing the functioning of their cognitive. 
Basing on the "use-it-or-lose-it" postulate [86] and on the ecological complexity premise[87], [85]suggested that inordinate network complexity thus being involved with diversity of social relationships or multiple roles in personal networks, could offers older adults with boosted intellectual engagement [88],[89], thereby stimulating neural activity and maintaining cognitive capabilities in the end thereafter..

In their investigation, it was found out those older persons recording greater numbers of association types in their network were characterized by advanced cognitive capabilities both in specific and global terms than their colleagues recording fewer types. Importantly, a reduction in the complexity of a network was correlated to deterioration in cognitive functioning. These outcomes were neither enlightened by magnitude of the personal network nor by existence of explicit relationship types in the network, which could ascertain the conception that complexity may possibly convey an added remunerations established on the machinery of stimulated brain stashes.

According to [85] there remained likewise suggestions for a cushioning outcome of network complexity on cognitive transformation above time. This outcome, nonetheless, existed non-considerably as dissimilarities in deterioration seemed to be somewhat negligible through diverse intensities or levels of network complexity. Still, the outcomes offered highlighted that $\mathrm{CN}$ with varied social relationships could perhaps add to enriched environments. These environments are cognitively challenging, they need exchanging and control between several settings, and expedite training of brain actions vital to neural plasticity $[87,90]$. It appears rewards are not only gained by incorporation into massive personal networks, but also by the incorporation into certain network structures.

Personal networks often change and shrink in future [91, 92] and such decrease in size of personal networks likely follows from deterioration in cognitive and physical functioning[93, 94].

[95] Recently used complex networks to address the issue of plagiarism. Plagiarism, a complex problem seen as the action of bestowing external wordings, ideas or thoughts, as persons own, without mention of the sources from which they were gotten. The progressive advancement of diverse digital document cradles existing on the Web has enabled the spread of the act, making its precise exposure a crucial task most especially for educational institutions. They proposed DOCODE 3.0; a Web system for educational establishments accomplishes automatic examination on huge quantities of digital documents relative to their degree of novelty. The system applies algorithms from multi data source to all these levels so as to execute an information fusion process.

A more recent study looked at how complex systems are applied in the field of metabolic engineering, explicitly taking into consideration the density of these networks. The literature [96] examined how complexity affects engineering at the protein, genome ranks, and passageway inside an organism, and the apparatuses for controlling these matters to achieve high-performing strain designs. Quantifiable complexity measures and their applicability to metabolic engineering as against traditional engineering disciplines remained also discussed. They further emphasized that, as metabolic engineering evolves into progressively standardized, complexity examination procedures and tools will remain an essential chunk of engineering toolbox.

\section{CONCLUSION AND RECOMMENDATION}

In any complex system, there occurs a network that describes the associations among the components. There are a massive number of systems that are despondently complex all over, ranging from the society, thus a set of six billion individuals to communication systems that connects billions of equipment together on the one side, to computers and cell phones on the other side. The very survival or existence of man is entrenched in the ability of thousands of genes to work in a seamless fashion. The systems which seemed random at a glimpse, exhibits infinite marks of order and self-organization at a close inspection but their complexity could actually be apprehended by carefully considering their fundamental constituents. Their quantification, understanding, forecast and the ultimate control is the foremost rational challenge for the science of the 21 st century. Human beings are bounded by complex networks hence need to apprehend the complexities involved and how they work

Although $\mathrm{CN}$ are not associated with any formal definition, its theory largely relies on graph theory for its topological structure, on probability theory to express distinguishing properties such as the degree and eigenvalue distributions and on dynamic systems theory to label the processes such as virus spread and synchronization on the network. However, there is still room for improvement as well as the necessity to carry out further research into its asymptotic scaling laws, topological metrics, new extremer graphs, and physical phenomena such as percolation, coupling, self-criticality, synchronization, emergent and cooperative behavior and other, commonly, nonlinear processes. Furthermore, there is opportunity for advance exploration in the area, most especially with the feature of the environment that inhibits these networks, structure and more so with concerns to control, synchronization and optimization.

\section{REFERENCES}

[1] A. Gasparri, S. Meloni, and S. Panzieri, "Growing Fully Distributed Robust Topologies in a Sensor Network," in Modelling, Estimation and Control of Networked Complex Systems, ed: Springer, 2009, pp. 143-158.

[2] L. A. Amaral and J. M. Ottino, "Complex networks," The European Physical Journal B-Condensed Matter and Complex Systems, vol. 38, pp. 147-162, 2004.

[3] C. Nan, I. Eusgeld, and W. Kröger, "Analyzing vulnerabilities between SCADA system and SUC due to interdependencies," Reliability Engineering \& System Safety, vol. 113, pp. 76-93, 2013.

[4] R. Allan, "Computational Research into Complex Systems," ed: Citeseer, 2009.

[5] Ş. Ş. Erçetin and S. M. Bisaso, "The Effect of Social Media and Social Networking on Perceptions of Leadership and Leaders," in Chaos, Complexity and Leadership 2014, ed: Springer, 2016, pp. 105-117.

[6] J. Kim and T. Wilhelm, "What is a complex graph?," Physica A: Statistical Mechanics and its Applications, vol. 387, pp. 2637-2652, 2008.

[7] A.-L. Barabási and E. Bonabeau, "Scale-free networks," Scientific American, vol. 288, pp. 50-59, 2003.

[8] D. J. Watts and S. H. Strogatz, "Collective dynamics of 'small-world'networks," nature, vol. 393, pp. 440-442, 1998. 
[9] L. A. N. Amaral, A. Scala, M. Barthelemy, and H. E. Stanley, "Classes of small-world networks," Proceedings of the national academy of sciences, vol. 97, pp. 1114911152, 2000.

[10] R. Albert and A.-L. Barabási, "Statistical mechanics of complex networks," Reviews of modern physics, vol. 74, p. $47,2002$.

[11] S. H. Strogatz, "Exploring complex networks," Nature, vol. 410, pp. 268-276, 2001.

[12] S. V. Buldyrev, R. Parshani, G. Paul, H. E. Stanley, and S. Havlin, "Catastrophic cascade of failures in interdependent networks," Nature, vol. 464, pp. 10251028,2010

[13] R. Parshani, S. V. Buldyrev, and S. Havlin, "Interdependent networks: Reducing the coupling strength leads to a change from a first to second order percolation transition," Physical review letters, vol. 105, p. 048701, 2010 .

[14] A. E. Motter and R. Albert, "in motion," Phys. Today, vol. 65 , p. 43, 2012.

[15] M. Abdulla, "On the fundamentals of stochastic spatial modeling and analysis of wireless networks and its impact to channel losses," Concordia University, 2012.

[16] B. Bollobás, "Random Graphs. 2001," Cambridge Stud. Adv. Math, 2001

[17] P. ERDdS and A. WI, "On random graphs I," Publ. Math. Debrecen, vol. 6, pp. 290-297, 1959.

[18] J. Bowen, "Hypercubes," Practical Computing, avril, 1982.

[19] P. G. GYARMA, "TI: SOME WORDS ABOUT NETWORKS."

[20] R. Cohen, K. Erez, D. Ben-Avraham, and S. Havlin, "Resilience of the Internet to random breakdowns," Physical review letters, vol. 85, p. 4626, 2000.

[21] R. Cohen and S. Havlin, "Scale-free networks are ultrasmall," Physical review letters, vol. 90, p. 058701, 2003

[22] P. Erd6s and A. Rényi, "On the evolution of random graphs," Publ. Math. Inst. Hungar. Acad. Sci, vol. 5, pp. 17-61, 1960.

[23] B. Bollobas, "Random Graphs, Academic," ed: London, 1985.

[24] M. Faloutsos, P. Faloutsos, and C. Faloutsos, "On powerlaw relationships of the internet topology," in ACM SIGCOMM computer communication review, 1999, pp. 251-262.

[25] H. Jeong, B. Tombor, R. Albert, Z. N. Oltvai, and A.-L. Barabási, "The large-scale organization of metabolic networks," Nature, vol. 407, pp. 651-654, 2000.

[26] J. Abello, A. L. Buchsbaum, and J. R. Westbrook, "A functional approach to external graph algorithms," in Algorithms-ESA'98, ed: Springer, 1998, pp. 332-343.

[27] A. Broder, R. Kumar, F. Maghoul, P. Raghavan, S. Rajagopalan, R. Stata, et al., "Graph structure in the web," Computer networks, vol. 33, pp. 309-320, 2000.
[28] A.-L. Barabási and R. Albert, "Emergence of scaling in random networks," science, vol. 286, pp. 509-512, 1999.

[29] A.-L. Barabási, R. Albert, and H. Jeong, "Mean-field theory for scale-free random networks," Physica A: Statistical Mechanics and its Applications, vol. 272, pp. 173-187, 1999.

[30] M. Boguná, R. Pastor-Satorras, and A. Vespignani, "Absence of epidemic threshold in scale-free networks with degree correlations," Physical review letters, vol 90, p. 028701, 2003.

[31] L. A. Nunes Amaral, A. Scala, M. Barthelemy, and H. E. Stanley, "Classes of behavior of small-world networks," arXiv preprint cond-mat/0001458, 2000.

[32] M. E. Newman, "The structure of scientific collaboration networks," Proceedings of the National Academy of Sciences, vol. 98, pp. 404-409, 2001.

[33] H. R. Bernard, P. D. Killworth, M. J. Evans, C. McCarty, and G. A. Shelley, "Studying social relations crossculturally," Ethnology, pp. 155-179, 1988.

[34] F. Karinthy, "Chain-links," Everything is different, pp 21-26, 1929.

[35] S. Milgram, "The small world problem," Psychology today, vol. 2, pp. 60-67, 1967.

[36] A. Wagner and D. A. Fell, "The small world inside large metabolic networks," Proceedings of the Royal Society of London B: Biological Sciences, vol. 268, pp. 1803-1810, 2001.

[37] L. Björneborn, "Small-world linkage and co-linkage," in Proceedings of the 12th ACM Conference on Hypertext and Hypermedia, 2001, pp. 133-137.

[38] B. Kogut and G. Walker, "The small world of Germany and the durability of national networks," American sociological review, pp. 317-335, 2001.

[39] O. Sporns, G. Tononi, and G. M. Edelman, "Theoretical neuroanatomy: relating anatomical and functional connectivity in graphs and cortical connection matrices," Cerebral Cortex, vol. 10, pp. 127-141, 2000

[40] K. E. Stephan, C. C. Hilgetag, G. A. Burns, M. A O'Neill, M. P. Young, and R. Kotter, "Computational analysis of functional connectivity between areas of primate cerebral cortex," Philosophical Transactions of the Royal Society B: Biological Sciences, vol. 355, pp. 111-126, 2000.

[41] J. M. Kleinberg, "Navigation in a small world," Nature, vol. 406, pp. 845-845, 2000

[42] J. Wallinga, W. J. Edmunds, and M. Kretzschmar, "Perspective: human contact patterns and the spread of airborne infectious diseases," Trends in microbiology, vol. 7, pp. 372-377, 1999.

[43] F. Ball, D. Mollison, and G. Scalia-Tomba, "Epidemics with two levels of mixing," The Annals of Applied Probability, pp. 46-89, 1997.

[44] M. J. Keeling, "The effects of local spatial structure on epidemiological invasions," Proceedings of the Royal Society of London B: Biological Sciences, vol. 266, pp. 859-867, 1999. 
[45] M. Boots and A. Sasaki, "'Small worlds' and the evolution of virulence: infection occurs locally and at a distance," Proceedings of the Royal Society of London B: Biological Sciences, vol. 266, pp. 1933-1938, 1999.

[46] L. F. Lago-Fernández, R. Huerta, F. Corbacho, and J. A. Sigüenza, "Fast response and temporal coherent oscillations in small-world networks," Physical Review Letters, vol. 84, p. 2758, 2000.

[47] M. Barthélémy and L. A. N. Amaral, "Small-world networks: Evidence for a crossover picture," Physical Review Letters, vol. 82, p. 3180, 1999.

[48] M. E. Newman, "Models of the small world," Journal of Statistical Physics, vol. 101, pp. 819-841, 2000.

[49] M. E. Newman, C. Moore, and D. J. Watts, "Mean-field solution of the small-world network model," Physical Review Letters, vol. 84, p. 3201, 2000.

[50] A. D. Barbour and G. Reinert, "Small worlds," Random Structures \& Algorithms, vol. 19, pp. 54-74, 2001.

[51] S. Boccaletti, V. Latora, Y. Moreno, M. Chavez, and D.U. Hwang, "Complex networks: Structure and dynamics," Physics reports, vol. 424, pp. 175-308, 2006.

[52] L. d. F. Costa, F. A. Rodrigues, G. Travieso, and P. R. Villas Boas, "Characterization of complex networks: A survey of measurements," Advances in Physics, vol. 56, pp. 167-242, 2007.

[53] P. Van Mieghem, Performance analysis of communications networks and systems: Cambridge University Press, 2006.

[54] E. R. Van Dam and W. H. Haemers, "Which graphs are determined by their spectrum?," Linear Algebra and its applications, vol. 373, pp. 241-272, 2003.

[55] P. Van Mieghem, Graph spectra for complex networks: Cambridge University Press, 2010.

[56] P. Van Mieghem, "The Physics of Complex Networks."

[57] S. N. Dorogovtsev, A. V. Goltsev, and J. F. Mendes, "Critical phenomena in complex networks," Reviews of Modern Physics, vol. 80, p. 1275, 2008.

[58] P. Van Mieghem, J. Omic, and R. Kooij, "Virus spread in networks," Networking, IEEE/ACM Transactions on, vol. 17, pp. 1-14, 2009.

[59] J. G. Restrepo, E. Ott, and B. R. Hunt, "Onset of synchronization in large networks of coupled oscillators," Physical Review E, vol. 71, p. 036151, 2005.

[60] S. H. Strogatz, "From Kuramoto to Crawford: exploring the onset of synchronization in populations of coupled oscillators," Physica D: Nonlinear Phenomena, vol. 143, pp. 1-20, 2000.

[61] P. Van Mieghem, C. Doerr, H. Wang, J. M. Hernandez, D. Hutchison, M. Karaliopoulos, et al., "A framework for computing topological network robustness," Delft University of Technology, Report20101218, 2010.

[62] Y. Chen, G. Paul, S. Havlin, F. Liljeros, and H. E. Stanley, "Finding a better immunization strategy," Physical review letters, vol. 101, p. 058701, 2008.

[63] J. Omić, J. Martín-Hernández, and P. Van Mieghem, "Network protection against worms and cascading failures using modularity partitioning," in Teletraffic Congress (ITC), 2010 22nd International, 2010, pp. 1-8.

[64] A. Arenas, A. Díaz-Guilera, J. Kurths, Y. Moreno, and C. Zhou, "Synchronization in complex networks," Physics Reports, vol. 469, pp. 93-153, 2008.

[65] N. Wiener, "Nonlinear problems in random theory. 1958," ed: New York.

[66] N. Wiener, "Cybernetics. 1961," First German edition, Duesseldorf, Econ, 1963.

[67] E. T. Hall, The dance of life: Anchor Press Garden City, NY, 1983.

[68] J. Pena Ramirez, "Huygens' synchronization of dynamical systems: beyond pendulum clocks," Technische Universiteit Eindhoven, 2013.

[69] A. T. Winfree, "Biological rhythms and the behavior of populations of coupled oscillators," Journal of theoretical biology, vol. 16, pp. 15-42, 1967.

[70] A. T. Winfree, The geometry of biological time vol. 12: Springer Science \& Business Media, 2001.

[71] J. Travers and S. Milgram, "An experimental study of the small world problem," Sociometry, pp. 425-443, 1969.

[72] X. F. Wang and G. Chen, "Complex networks: smallworld, scale-free and beyond," Circuits and Systems Magazine, IEEE, vol. 3, pp. 6-20, 2003.

[73] L. Zhao, Y.-C. Lai, K. Park, and N. Ye, "Onset of traffic congestion in complex networks," Physical Review E, vol. 71, p. 026125, 2005.

[74] C.-Y. Yin, W.-X. Wang, G. Chen, and B.-H. Wang, "Decoupling process for better synchronizability on scale-free networks," Physical Review E, vol. 74, p. 047102, 2006.

[75] P. Van Mieghem, D. Stevanović, F. Kuipers, C. Li, R. Van De Bovenkamp, D. Liu, et al., "Decreasing the spectral radius of a graph by link removals," Physical Review E, vol. 84, p. 016101, 2011

[76] F. A. Kuipers and P. F. Van Mieghem, "Conditions that impact the complexity of QoS routing," IEEE/ACM Transactions on Networking (TON), vol. 13, pp. 717 $730,2005$.

[77] F. Kuipers, A. Beshir, A. Orda, and P. Van Mieghem, "Impairment-aware path selection and regenerator placement in translucent optical networks," in Network Protocols (ICNP), 2010 18th IEEE International Conference on, 2010, pp. 11-20.

[78] P. Van Mieghem and L. Vandenberghe, "Trade-Off Curves for QoS Routing," in INFOCOM, 2006.

[79] S. Tang, E. Jaho, I. Stavrakakis, I. Koukoutsidis, and P. Van Mieghem, "Modeling gossip-based content dissemination and search in distributed networking," Computer Communications, vol. 34, pp. 765-779, 2011.

[80] A. Czumaj and C. Sohler, "Sublinear-time algorithms Bulletin of the EATCS, 89: 23 47," ed: June, 2006.

[81] C. J. Stam and J. C. Reijneveld, "Graph theoretical analysis of complex networks in the brain," Nonlinear biomedical physics, vol. 1, p. 3, 2007. 
[82] C. J. Stam, A. Hillebrand, H. Wang, and P. Van Mieghem, "Emergence of modular structure in a largescale brain network with interactions between dynamics and connectivity," Frontiers in computational neuroscience, vol. 4, 2010.

[83] M. P. van den Heuvel, C. J. Stam, R. S. Kahn, and H. E. H. Pol, "Efficiency of functional brain networks and intellectual performance," The Journal of Neuroscience, vol. 29, pp. 7619-7624, 2009.

[84] H. Wang, L. Douw, J. M. Hernandez, J. Reijneveld, C. Stam, and P. Van Mieghem, "Effect of tumor resection on the characteristics of functional brain networks," Physical Review E, 82 (2), 2010, 2010.

[85] L. Ellwardt, T. G. Van Tilburg, and M. J. Aartsen, "The mix matters: Complex personal networks relate to higher cognitive functioning in old age," Social Science \& Medicine, vol. 125, pp. 107-115, 2015.

[86] D. F. Hultsch, C. Hertzog, B. J. Small, and R. A. Dixon, "Use it or lose it: engaged lifestyle as a buffer of cognitive decline in aging?," Psychology and aging, vol. 14, p. 245, 1999.

[87] C. Schooler, Psychological effects of complex environments during the life span: A review and theory: Ablex Publishing, 1987.

[88] C. Stephens, F. Alpass, A. Towers, and B. Stevenson, "The effects of types of social networks, perceived social support, and loneliness on the health of older people: Accounting for the social context," Journal of Aging and Health, p. 0898264311400189, 2011.

[89] L. C. Giles, K. J. Anstey, R. B. Walker, and M. A. Luszcz, "Social networks and memory over 15 years of followup in a cohort of older Australians: results from the Australian Longitudinal Study of Ageing," Journal of aging research, vol. 2012, 2012.
[90] M. J. Valenzuela, F. E. Matthews, C. Brayne, P. Ince, G. Halliday, J. J. Kril, et al., "Multiple biological pathways link cognitive lifestyle to protection from dementia," Biological psychiatry, vol. 71, pp. 783-791, 2012.

[91] M. B. van Groenou, E. O. Hoogendijk, and T. G. van Tilburg, "Continued and New Personal Relationships in Later Life Differential Effects of Health," Journal of aging and health, vol. 25, pp. 274-295, 2013.

[92] C. Wrzus, M. Hänel, J. Wagner, and F. J. Neyer, "Social network changes and life events across the life span: A meta-analysis," Psychological Bulletin, vol. 139, p. 53, 2013.

[93] M. J. Aartsen, C. H. Smits, T. van Tilburg, K. C. Knipscheer, and D. J. Deeg, "Activity in older adults cause or consequence of cognitive functioning? A longitudinal study on everyday activities and cognitive performance in older adults," The Journals of Gerontology Series B: Psychological Sciences and Social Sciences, vol. 57, pp. P153-P162, 2002.

[94] M. J. Aartsen, T. Van Tilburg, C. H. Smits, and K. C. Knipscheer, "A longitudinal study of the impact of physical and cognitive decline on the personal network in old age," Journal of Social and Personal Relationships, vol. 21, pp. 249-266, 2004.

[95] J. D. Velásqueza, Y. Covacevicha, F. Molinaa, E. Marrese-Taylora, C. Rodrígueza, and F. BravoMarquezb, "DOCODE 3.0 (DOcument COpy DEtector): A system for plagiarism detection by applying an information fusion process from multiple documental data sources."

[96] J. D. Winkler, K. Erickson, A. Choudhury, A. L. Halweg-Edwards, and R. T. Gill, "Complex systems in metabolic engineering," Current opinion in biotechnology, vol. 36, pp. 107-114, 2015. 